\title{
Análises pelos discentes de uma escola médica das atividades de integração ensino-serviço na graduação
}

\author{
Analysis of Service-learning activities by a medical school's learners \\ Análisis de las actividades de integración enseñanza-servicio por lor esdudiantes de uma \\ escuela medica
}

Santino Carvalho Franco ${ }^{1 *}$, Deborah Holanda da Silva Brayde ${ }^{1}$, Deivid Ramos dos Santos², José Antônio Cordero².

\section{RESUMO}

Objetivo: descrever a percepção dos discentes quanto ao eixo de práticas de integração ensino-serviço na Atenção Básica em um curso de Medicina. Métodos: Trata-se de uma pesquisa quantitativa e descritiva, que ocorreu na Faculdade Metropolitana da Amazônia, com discentes do eixo de Interação, ensino, serviço, comunidade e gestão, através de questionário com perguntas sócio demográficas e com base nas principais recomendações das Diretrizes Curriculares Nacionais dos cursos de Medicina, utilizando-se a escala Likert. Resultados: Para todas as questões as respostas obtidas foram "concordo totalmente" e "concordo parcialmente" em sua maioria, como na contribuição da consolidação do SUS, na compreensão da integralidade do cuidado e no desenvolvimento do trabalho em equipe. Todas as questões tiveram Ranking Médio acima de 4. Conclusão: vale destacar que as Diretrizes Nacionais Curriculares, ratificam a importância da formação generalista e a capacidade de mobilizar conhecimentos para qualificar a prática profissional. Porém, há necessidade de novos estudos com vistas a aprofundar a compreensão da integração ensinoserviço no contexto da Atenção Básica.

Palavras-chave: Atenção Primária em Saúde, Educação Médica, Estratégia Saúde da Família.

\begin{abstract}
Objective: Describe the perception of students in relation to the practices of integration of service-teaching in Basic Attention in a Medicine course. Methods: It is a quantitative and descriptive research, achieved in Faculdade Metropolitana da Amazônia, with students from Integration, teaching, service, community and management, through a questionnaire of sociodemographic questions and based in the main recommendation of the National Curricular Guidelines of Medicine courses, using the Likert scale for evaluation and the average ranking to analize the data. Results: The results came from the answers totally agree and partially agree, in its majority, in relation to the contribution of SUS's consolidation, to the understanding of care integrality and to the development of team work. All questions obtained average ranking over 4. Conclusion: The National Curricular Guidelines certify the importance of general formation and the capability of mobilaizing knowledge to qualify the professional practice. However, there is the necessity of new studies to deep in the comprehension of integration service-teaching, in the aspect of Basic Atention.
\end{abstract}

Keywords: Basic Attention to Health, Medical Education, Family Health Strategy.

\section{RESUMEN}

Objetivo: Describir la percepción de los discentes cuanto al eje de prácticas de integración enseñanzaservicio en la Atención Básica en un curso de Medicina. Métodos: Tratase de una pesquisa cuantitativa y descriptiva, que ocurrió en la Facultad Metropolitana de la Amazonia, con los discentes del eje de Integración, enseñanza, servicio, comunidad y gestión, por medio de cuestionario con preguntas sociodemográficas y con base en las principales recomendaciones de las Directrices Curriculares Nacionales de los cursos de

${ }^{1}$ Centro Universitário Metropolitano da Amazônia (UNIFAMAZ), Belém- PA.

*E-mail: deivid_ramos45@hotmail.com

2 Universidade do Estado do Pará (UEPA), Belém - PA. 
Medicina, utilizándose la escala Likert. Resultados: Los resultados obtenidos fueron respuestas "de acuerdo totalmente" y "de acuerdo parcialmente", en su mayoría como contribución en la consolidación del SUS, en la comprensión de la integralidad del cuidado y en el desarrollo del trabajo en equipo. Todas las cuestiones tuvieron Ranking Medio arriba de 4. Conclusión: Merece destacar que las Directrices Nacionales Curriculares, ratifican la importancia de la formación generalista y la capacidad de movilizar conocimientos para calificar la práctica profesional. Pero, hay la necesidad de nuevos estudios con el objetivo de ahondar la comprensión de la integración enseñanza-servicio en el contexto de la Atención Básica.

Palabras clave: Atención Primaria en Salud, Educación Médica, Estrategia Salud de la Familia.

\section{INTRODUÇÃO}

Em 1987, a Organização Mundial de Saúde (OMS), recomendou reformas nos currículos das universidades que possuem cursos da área da saúde e dentre as mudanças na metodologia de ensino estava o alinhamento da educação em saúde de acordo com as necessidades da comunidade. Diante disso, no Reino Unido, esse alinhamento das escolas médicas de ensino baseado em serviço na comunidade trouxe ganhos significativos para o aprendizado dos estudantes (LEE GG e ONG CP, 2014).

Nesse sentido, pesquisadores têm demonstrado a relação positiva entre educação e serviços de saúde, sendo que essa integração é similar ao que acontece em diversos programas nacionais, como o programa Saúde na Escola, em que políticas públicas e práticas associadas contribuem para melhora na qualidade do ensino e de assistência à população (CHIANG RJ et al, 2015).

Além disso, é fundamental a identificação de fatores que dificultam o ensino e a aprendizagem dos alunos. Entre eles, podemos ter fatores motivacionais, problemas em se adequar ao aprendizado sem a ação direta do professor, pressões familiares e estresse (GOH LH e ONG CP, 2014).

A partir de tudo que foi exposto, há algumas competências que a integração ensino-serviço também objetiva inserir na atitude do futuro profissional, ente elas temos: a medicina centrada na pessoa; o cuidado contínuo e vínculo com o paciente, além de promoção da saúde; aprimoramento do conhecimento médico aplicado nesse cuidado; aprendizado baseado na prática e embasado nas melhores evidências científicas; habilidade de comunicação com os pacientes, seus familiares e a comunidade, bem como o trabalho multiprofissional; desenvolver atenção à saúde e ensino conjuntamente; e responsabilidade profissional, primando sempre pelos princípios éticos (GOH LH e ONG CP, 2014).

Com tudo isso, foram definidas as Diretrizes Curriculares Nacionais de Medicina em 2014 que preconizam a aquisição, durante a graduação, de competências e habilidades gerais que se referem à tomada de decisão, comunicação, trabalho em equipe, liderança, gerenciamento e educação permanente e, além disso, incentivam modelos de ensino médico nos quais os estudantes devem estar envolvidos, desde os primeiros períodos, em atividades curriculares ligadas à promoção, à prevenção, à reabilitação e à recuperação da saúde. Isso evidencia a necessária articulação entre conhecimentos, habilidades e atitudes requeridas para o exercício profissional do médico nas áreas de Atenção à Saúde, Gestão em Saúde e Educação em Saúde.

Dessa forma, o presente estudo teve o objetivo de descrever a percepção dos discentes quanto às contribuições do eixo integração ensino e serviço para a formação médica.

\section{MÉTODOS}

Trata-se de um estudo de campo analítico, descritivo e exploratório, com abordagem quantitativa. A pesquisa foi realizada na UNIFAMAZ, com 83 alunos do Curso de Medicina que frequentam as Unidades de Saúde da Família (USF) do Município de Belém/PA, as quais, recebem alunos do eixo Interação, ensinoserviço, comunidade e gestão (IESCG), em seus $7^{\circ}$ e $8^{\circ}$ períodos, no período de maio de 2018.

A pesquisa de campo compreendeu a coleta de dados através de questionário contendo questões socioeconômicas com 6 perguntas semiestruturadas fechadas e a segunda parte com 12 perguntas semiestruturadas abertas, com base nas principais recomendações das Diretrizes Curriculares Nacionais do Curso de Medicina de 2014. 
Para análise dos itens utilizou-se o cálculo do Ranking Médio (RM). Neste modelo atribui-se um valor de 1 a 5 para cada resposta a partir da qual é calculada a média ponderada para cada item, baseando-se na frequência das respostas. Quanto mais próximo de 5 , maior será o nível de satisfação dos estudantes e quanto mais próximo de 1 , menor a satisfação. As tabelas foram construídas com auxílio do programa Microsoft Office 2018 ®.

A pesquisa respeita os preceitos da Declaração de Helsinque e do Código de Nuremberg, conforme o Conselho Nacional de Saúde, 466 de 2012. Este trabalho foi aprovado sob o parecer 2.631.725 no Comitê de Ética em Pesquisa da UNIFAMAZ.

\section{RESULTADOS}

Como resultados deste estudo, pode-se observar a distribuição dos participantes conforme o sexo, faixa etária, naturalidade, se trabalha ou não, se possui outra formação e o período que está cursando. $63,88 \%$ $(n=53)$ são mulheres, $41 \%(n=34)$ possui idade entre 18 a 25 anos, 56,7\% $(n=47)$ não é natural de Belém, $89,16 \%$ (74) não trabalha e 18,07\% ( $n=15)$ possuem outro curso de nível superior (Tabela 1).

Tabela 1 - Características socioeconômico dos discentes dos $7^{\circ}$ e 8ํㅜ períodos do curso de Medicina da UNIFAMAZ, Belém, Pará, Brasil, 2019.

\begin{tabular}{|c|c|c|c|c|}
\hline & VARIÁVEIS & & $\mathbf{N}$ & $\%$ \\
\hline \multirow{2}{*}{ Q1 } & SEXO & Masculino & 30 & 36,15 \\
\hline & \multirow{4}{*}{ FAIXA ETÁRIA } & Feminino & 53 & 63,85 \\
\hline \multirow{3}{*}{ Q2 } & & $18-25 a$ & 34 & 40,97 \\
\hline & & $26-30 \mathrm{a}$ & 43 & 51,8 \\
\hline & & $31-40 a$ & 5 & 6.03 \\
\hline \multirow{3}{*}{ Q3 } & \multirow{3}{*}{ NATURALIDADE } & $>40$ anos & 1 & 1,2 \\
\hline & & Belém & 36 & 43,37 \\
\hline & & Outra & 47 & 56,63 \\
\hline \multirow{2}{*}{ Q4 } & \multirow{2}{*}{ TRABALHA } & Sim & 9 & 10,84 \\
\hline & & Não & 74 & 89,16 \\
\hline \multirow{2}{*}{ Q5 } & \multirow{2}{*}{ OUTRA FORMAÇÃO } & Sim & 15 & 18,07 \\
\hline & & Não & 68 & 81,93 \\
\hline \multirow{3}{*}{ Q6 } & DERínn & $7^{0}$ & 36 & 43,37 \\
\hline & PENIUUV & $8^{\circ}$ & 47 & 56,63 \\
\hline & TOTAL & & 83 & $100 \%$ \\
\hline
\end{tabular}

Fonte: Protocolo de pesquisa, 2019. Q: questão.

Como visto na Tabela 2, a percepção dos alunos conforme ranking médio. $O$ grau de satisfação médio ficou entre 4 e 5 pontos. Em nenhuma das questões houve alunos que discordaram parcialmente e pequena parcela discordou totalmente (aproximadamente 5\%). 
Tabela 2 - Ranking Médio da percepção dos discentes quanto à Integração ensino e serviço na comunidade, Belém, Pará, Brasil, 2019.

\begin{tabular}{|c|c|c|c|c|c|c|c|}
\hline & QUESTÕES & $\begin{array}{c}\text { DP } \\
\text { n (\%) }\end{array}$ & $\begin{array}{c}\text { DT } \\
\text { n (\%) }\end{array}$ & $\begin{array}{c}\text { NC/ND } \\
n(\%)\end{array}$ & $\begin{array}{c}\mathrm{CP} \\
\mathrm{n}(\%)\end{array}$ & $\begin{array}{c}\text { CT } \\
\text { n (\%) }\end{array}$ & $\begin{array}{l}\text { RANKING } \\
\text { MÉDIO }\end{array}$ \\
\hline Q7 & $\begin{array}{lr}\text { Contribui para } & 0 \\
\text { desenvolvimento } & \text { do } \\
\text { trabalho em equipe. } & \end{array}$ & - & $3(3,62)$ & $7(8,43)$ & $27(32,53)$ & $\begin{array}{c}46(55,42 \\
)\end{array}$ & 4,39 \\
\hline Q8 & $\begin{array}{l}\text { O trabalho desenvolvido } \\
\text { pelo serviço contribui para } \\
\text { o estudante compreender a } \\
\text { proposta do SUS. }\end{array}$ & - & $3(3,62)$ & $7(8,43)$ & $21(25,2)$ & $\begin{array}{c}52(62,75 \\
)\end{array}$ & 4,51 \\
\hline Q9 & $\begin{array}{l}\text { Possibilita contemplar a } \\
\text { participação de todos os } \\
\text { profissionais da equipe. }\end{array}$ & - & $2(2,41)$ & $15(18,7)$ & $8(9,64)$ & $\begin{array}{c}58(68,88 \\
)\end{array}$ & 4,46 \\
\hline Q10 & $\begin{array}{l}\text { Possibilita a produção e } \\
\text { divulgação do } \\
\text { conhecimento voltado } \\
\text { para atenção básica. }\end{array}$ & - & $2(2,41)$ & $8(9,64)$ & $25(30,12)$ & $\begin{array}{c}48(57,83 \\
)\end{array}$ & 4,43 \\
\hline Q11 & $\begin{array}{l}\text { Contribui para a } \\
\text { compreensão da } \\
\text { integralidade do cuidado. }\end{array}$ & - & - & $3(3,62)$ & $25(30,12)$ & $\begin{array}{c}55(66,26 \\
)\end{array}$ & 4,62 \\
\hline Q12 & $\begin{array}{l}\text { Favorece a educação } \\
\text { permanente dos } \\
\text { profissionais de saúde. }\end{array}$ & - & - & $6(7,23)$ & $33(39,76)$ & $\begin{array}{c}44(53,01 \\
)\end{array}$ & 4,45 \\
\hline Q13 & $\begin{array}{l}\text { Contribui para que a equipe } \\
\text { conheça melhor as } \\
\text { necessidades das pessoas, } \\
\text { das famílias e da } \\
\text { comunidade }\end{array}$ & - & $1(1,20)$ & $7(8,43)$ & $20(24,1)$ & $\begin{array}{c}55(66,27 \\
)\end{array}$ & 4,55 \\
\hline Q14 & $\begin{array}{lr}\begin{array}{l}\text { Desenvolve ações } \\
\text { outros setores }\end{array} \\
\text { comunidade, da } \\
\text { escolas, setor } \\
\text { urbanização e outros. }\end{array}$ & - & $1(1,20)$ & $16(87)$ & $35(42,17)$ & $\begin{array}{c}33(39,76 \\
)\end{array}$ & 4,2 \\
\hline Q15 & $\begin{array}{l}\text { Favorece a compreensão } \\
\text { do contexto social. }\end{array}$ & - & $2(2,41)$ & $8(9,64)$ & $30(36,15)$ & $43(51,8)$ & 4,37 \\
\hline Q16 & $\begin{array}{l}\text { Contribui para a mudança } \\
\text { das práticas profissionais e } \\
\text { da } \\
\text { organização do trabalho. }\end{array}$ & - & $4(4,82)$ & $6(7,23)$ & $34(42,17)$ & $\begin{array}{c}37(45,78 \\
)\end{array}$ & 4,18 \\
\hline Q17 & $\begin{array}{l}\text { Contribui para a } \\
\text { consolidação do Sistema } \\
\text { Único de Saúde. }\end{array}$ & - & $4(4,82)$ & $\begin{array}{c}10(12,05 \\
)\end{array}$ & $28(33,73)$ & $41(49,4)$ & 4,27 \\
\hline Q18 & $\begin{array}{l}\text { Torna possível a } \\
\text { consolidação do controle } \\
\text { social. }\end{array}$ & - & $3(3,62)$ & $\begin{array}{c}11(13,25 \\
)\end{array}$ & $28(33,73)$ & $41(49,4)$ & 4,28 \\
\hline
\end{tabular}

Legenda: DT: discordo totalmente; DP: discordo parcialmente; NC/ND: nem condordo/nem discordo; $\mathrm{CP}$ : concordo parcialmente; CT: concordo totalmente. Q: questão.

Fonte: Protocolo de pesquisa, 2019. 


\section{DISCUSSÃO}

É possível observar na Tabela 01 os dados sociodemográficos dos discentes. Notou-se que a maioria dos alunos é do sexo feminino $(63,85 \%, n=53)$. Tal aspecto corrobora com o aumento do número de mulheres formadas em Medicina a partir do ano de 2000, como demonstrado em algumas pesquisas (PICOLI RP et al., 2017; SCHEFFER M et al., 2011).

A maioria dos alunos tem idade menor que 30 anos de idade, de localidade diferente da faculdade e não trabalham (Tabela 01). Resultados semelhantes foram encontrados em trabalho sobre o perfil de discentes de Medicina no Espírito Santo, neste estudo predominaram jovens de até 24 anos (76,4\%), seguindo a mesma tendência nacional (FIOROTTI KP et al., 2010; MACHADO MFAS et al.,2015).

Ainda nessa pesquisa, destaca-se que $54,2 \%$ dos discentes de Medicina da Universidade Estadual do Rio Grande do Norte (UERN) é nascida em cidades do interior do Estado onde estudam e apenas 20,8\% moram com os pais, situação também observada neste trabalho. Isso se deve à concentração dos cursos de Medicina estarem em regiões metropolitanas, o que obriga o aluno a buscar a graduação fora de sua cidade de origem em direção à capital (MARIN MJS et al.,2014).

Com relação ao exercício de alguma atividade remunerada, 89,16\% ( $n=74)$ afirmaram que não trabalham. Isso vai ao encontro dos achados que mostra a dependência financeira de grande parcela do alunado de Medicina. A necessidade de tempo para estudar para a graduação em medicina e a exigência do curso faz com que seja imprescindível o suporte familiar (MARIN MJS et al.,2014). No universo desta pesquisa, a maioria dos alunos pesquisados não tem formação anterior, porém $18,7 \%(n=15)$ já são graduados em outra área. Os principais motivos que levam os alunos a buscar outra graduação são: insatisfação com a profissão anterior, desejo antigo de ser médico, procura por melhores remunerações e mercado de trabalho (CORREA $\mathrm{RD}$ et al.,2016).

Os discentes, em sua maioria, concordaram que as atividades contemplam a participação de todos os profissionais da equipe de saúde. A atuação da equipe multidisciplinar não está relacionada à diversidade e/ou à quantidade de profissionais em um mesmo espaço, mas à capacidade de apreender a complexidade dos problemas em saúde, os quais, na maioria das vezes, estão associados a múltiplos fatores e causas, sendo necessário de serem apreendidos e respondidos por diferentes profissionais. É imprescindível a necessidade das dos alunos e funcionários do campo de prática refletirem sobre o seu trabalho, otimizando o processo de integração, pois isso impacta na compreensão dos projetos do serviço, do ensino e de organização das comunidades (FARIA L al.,2018; FEUERWERKER L et al., 2003).

Nas últimas décadas, os Ministérios da Saúde e da Educação apostaram em ações que favorecem a reorientação da formação na área da saúde. Entre elas, o Programa Nacional de Reorientação da Formação Profissional em Saúde (PróSaúde), lançado em 2005, que busca incentivar a transformação do processo de formação, geração de conhecimento e prestação de serviços à população, a fim de incorporar uma abordagem integral ao cuidado em saúde, principalmente voltado para o SUS (VENDRUSCOLO $\mathrm{C}$ et al., 2016; MARIN MJS et al., 2014).

A maioria concorda que as atividades possibilitaram a produção e divulgação de conhecimento voltado para a atenção básica. Observou-se em estudo de que as áreas da educação e da saúde vêm propondo relações de parceria entre universidades e serviços como forma de garantir investimentos no aperfeiçoamento dos profissionais, uma estrutura acadêmica apoiada em relações de parceria da universidade com os diversos setores da sociedade. A integração ensino-serviço deve ser um alicerce da construção de um novo modo de ensinar, aprender e fazer, efetivo para todos os sujeitos envolvidos: docentes, discentes, gestores das Instituições de ensino superior (IES) e do SUS, profissionais e população (VENDRUSCOLO et al., 2016).

Em relação à contribuição da integralidade do cuidado, houve uma boa avaliação. Em articulação com o sistema de saúde vigente, um conceito recorrente no discurso das Diretrizes Curriculares Nacionais (DCN) dos Cursos de Graduação em Saúde é o da integralidade, considerado um termo ou se apresentando como competência e habilidades: prática profissional de forma integrada; planejamento de intervenções de 
promoção, prevenção e reabilitação à saúde configurando atenção integral aos indivíduos, famílias e comunidades; responsabilidade pela qualidade da assistência/cuidado na perspectiva da integralidade da assistência (PIMENTEL EC et al.,2015).

Também foi positiva a percepção com relação à educação permanente dos profissionais de saúde. Para a educação permanente é outra competência de destaque na formação, visto que incentiva o profissional de saúde a desenvolver elevado grau de crítica, capacidade de compreender as necessidades da sociedade e de si, e o desenvolvimento de autonomia e de incremento coletivo (PICOLI RP et al., 2017; MAKUCH DMV et al.,2017).

Em estudo da Pontifícia Universidade Católica do Rio Grande do Sul(PUC-RS), foi elaborada estratédia de educação em saúde por meio de seminários itinerantes, cujo foco foi o conjunto de profissionais atuantes nas Unidades Básicas de Saúde (UBS) e da Estratégia de Saúde da Família (ESF), tendo como principal objetivo a promoção de discussões sobre o desenvolvimento de ações em saúde na região (PIZZINATO A et al., 2012).

Muitas experiências reconhecem os cenários de prática na rede básica como espaços onde as pessoas vivem, trabalham possibilidades, ensinam, aprendem e se organizam frente aos múltiplos determinantes da saúde. Nesses espaços se oferecem oportunidades de desenvolver e aperfeiçoar habilidades de cuidado, em situações reais do trabalho e da população, com a realização de práticas assistenciais junto a indivíduos, famílias, grupos e comunidade. Logo, há possibilidade de troca de conhecimentos entre estudantes, equipe e população. Na integração ensino-serviço, o paciente sente-se melhor acolhido pelo contato e receptividade dos estudantes, que identificam conflitos e constroem planos para as necessidades individuais e coletivas da área adstrita à unidade de saúde (KUABARA CTM et al.,2016).

Segundo as Diretrizes Curriculares Nacionais (DCN) do curso de Medicina permite a aproximação gradativa dos estudantes com a rede de Atenção Primária à Saúde do município, valorizando os saberes populares e comunitários presentes no território (GONDIM GM e MONKEN M, 2016; SILVIA AB et al.,2014; MACHADO MFAS et al., 2015). É fundamental o engajamento quanto ao papel social do médico, e deve ser estimulada desde o início do curso, por mais que não sejam completamente desenvolvidas. Se não houver, desde os primeiros semestres da graduação, o indicativo da importância do trabalho dentro da comunidade, sendo responsável socialmente, há grandes possibilidades de o estudante não conseguir realizar essas ações após a graduação, pois seu olhar poderá estar limitado à medicina estritamente curativa (FRANCO ML, 2005; BRIXNER B et al., 2017).

Nesse sentido, ao inserir os alunos à rotina de prática médica, a faculdade possibilita o desenvolvimento de uma postura diferenciada, tendo como fruto a complexidade do cuidado que exige não apenas conhecimentos técnicos, mas o afetivo diante de diferentes realidades sociais, muitas das vezes, de pessoas vulneráveis (VASCONCELOS ACF et al., 2016).

Dessa forma, a tríade formada pela universidade, serviços de saúde e a comunidade, tem papel imperioso na formação do aluno em relação à realidade de saúde na qual a população está inserida e seus respectivos anseios e necessidades (VASCONCELOS ACF et al., 2016; MARIN MJS et al., 2014).

Por outro lado, uma parcela dos alunos não observa essas mudanças. Em estudo com os gestores de Secretarias de Saúde do Rio de Janeiro (RJ), as principais dificuldades relacionadas aos recursos humanos, citam a deficiência no processo de formação, que ainda não prepara o profissional para atuar no âmbito da Atenção Básica, especialmente o médico. $E$, nesse sentido, o município tem investido não somente na aproximação da rede de saúde com a universidade, mas também na qualificação da sua força de trabalho, disponibilizando cursos técnicos, de especialização e mestrado. Também como dificuldade no processo de gestão do trabalho, há a percepção de que os profissionais e usuários ainda relutam em compreender e aceitar a mudança do modelo de gestão, de um centrado no médico, com foco na doença e organizado por demanda espontânea, para um que se alicerça na equipe multiprofissional e nas ações de caráter de promoção e prevenção de saúde (MACNAGO C e PIERANTONI CR, 2015; KUABARA CTM et al., 2016). 
Quanto à análise da tabela 01, todas as questões tiveram Ranking Médio acima de 4. É importante destacar que a avaliação positiva delas pode estar relacionada com a organização curricular do curso, que contempla o método pedagógico Aprendizagem Baseada em Problemas (ABP) e o ensino orientado para a comunidade, sendo a Estratégia Saúde da Família (ESF) um relevante cenário de aprendizagem. Além disso, sinaliza, de modo indireto, que a organização do curso, alicerçada na educação por competências, sugere a superação da visão tecnicista e o estímulo à capacidade de identificar as necessidades de resolver os problemas de saúde (PORTELLA MB et al., 2016; SHIMIZU HE e MOURA LM et al.,2015)

Nesse sentido, vale destacar que a DNC, ratificam a importância da formação generalista e a capacidade de mobilizar conhecimentos para qualificar a prática profissional (BRASIL, 2014).

\section{CONSIDERAÇÕES FINAIS}

$\mathrm{Na}$ análise geral, as avaliações foram positivas com relação às percepções e as contribuições da integração ensino-serviço, ao possibilitar a inserção precoce do estudante no contexto do trabalho da Atenção Básica e o processo de ensino e aprendizagem a partir da prática profissional vivenciada no cotidiano desses serviços. Há necessidade de diferentes e maiores abordagens com relação à compreensão da proposta do SUS, mais atividades que envolvam toda a equipe de saúde e ações que aproximem os alunos da comunidade para conhecer melhor a realidade das áreas adstritas às Unidades em Saúde da Família.

\section{REFERÊNCIAS}

1. BRASIL. Diretrizes Curriculares Nacionais do Curso de Medicina. Conselho Nacional de Educação. Câmara de Educação Superior. Resolução nํㅜ 3, de 20 de junho de 2014.

2. BRIXNER B et al. Ações de promoção de saúde nas estratégias saúde da família. Cinergis, Santa Cruz do Sul, 18(1); 386-390, 2017.

3. CARDOSO FAB et al. Perfil do Estudante de Medicina da Universidade do Estado do Rio Grande do Norte (UERN), 2013. REVISTA BRASILEIRA DE EDUCAÇÃO MÉDICA. 39 (1): 32-40; 2015.

4. CORREA RD et al. Medicina como Nova Graduação: Motivações, Dificuldades e Expectativas. REVISTA BRASILEIRA DE EDUCAÇÃO MÉDICA. 40(2);226-233, 2016.

5. CHIANG RJ et al. How the Whole School, Whole Community, Whole Child model works: creating greateralignment, integration, and collaboration between health and education. J Sch Health. 85(0), p.775-784, 2015

6. FARIA L et al. Integração ensino-serviço-comunidade nos cenários de práticas na formação interdisciplinar em Saúde: uma experiência do Programa de Educação pelo Trabalho para a Saúde (PET-Saúde) no sul da Bahia, Brasil. Interface - Comunicação, Saúde , Educação. 22 (67);1257-66,2018.

7. FEUERWERKER L. Educação dos profissionais de saúde hoje: problemas, desafios, perspectivas e as propostas do Ministério da Saúde. Revista da ABENO. 3(1); 24-27, 2003.

8. FIOROTTI KP et al. Perfil do Estudante de Medicina da Universidade Federal do Espírito Santo, 2007. REVISTA BRASILEIRA DE EDUCAÇÃO MÉDICA. 34 (3); 355 - 362, 2010.

9. FRANCO ML. Análise de conteúdo. Série pesquisa. Brasília: Líber Livro, 2005

10. GOH LG, ONG CP. Education and training in family medicine: progress and a proposed national vision for 2030 . Singapore Med J. 55(3); 117-123, 2014.

11. GONDIM GM, MONKEN M. Territorialização em saúde. In: Pereira IB, Lima JCF. Dicionário da educação profissional em saúde [Internet]. 2a ed. Rio de Janeiro: EPSJV. P.392-9. 2008.

12. KUABARA CTM et al. Integração ensino-serviço na perspectiva dos profissionais da rede primária de saúde. Revista Fórum Identidades. 22 (22), jan./abr, 173-192, 2016.

13. LEE GG, ONG CP. Education and training in family medicine: progress and a proposed national vision for 2030. Singapore Med J. 3 (55); 117-123, 2014.

14. MACHADO MFAS et al. Programa Saúde na Escola: estratégia promotora de saúde na atenção básica no Brasil. J. Hum. Growth Dev. 25 (3); 307-312, 2015.

15. MAGNAGO C, PIERANTONI CR. Dificuldades e estratégias de enfrentamento referentes à gestão do trabalho na Estratégia Saúde da Família, na perspectiva dos gestores locais: a experiência dos municípios do Rio de Janeiro (RJ) e Duque de Caxias (RJ). SAÚDE DEBATE. RJ,39(104);9-17, JAN-MAR 2015.

16. MAKUCH DMV et al. A integralidade do Cuidado no Ensino na Área da Saúde: uma Revisão Sistemática. Revista Brasileira de Educação Médica. 41(4); 515-524, 2017.

17. MARIN MJS et al. A integração ensino-serviço na formação de enfermeiros e médicos: a experiência da FAMEMA. Ciência \& Saúde Coletiva, 19 (3);967-974, 2014.

18. MIRANDA GMD et al. O desafio da organização do Sistema Único de Saúde universal e resolutivo no pacto federativo brasileiro. Saúde Soc. São Paulo, 26(2); 329-335, 2017. 
19. PíCOLI RP et al. Competências Propostas no Currículo de Medicina: Percepção do Egresso. Revista Brasileira de Educação Médica. 41(3);364-371; 2017.

20. PIMENTEL EC et al. Ensino e aprendizagem em estágio supervisionado: Estágio integrado em saúde. Rev Bras Educ Méd. 39(0); 3352-358, 2015.

21. PORTELLA MB et al. Projeto Político Pedagógico do Curso de Medicina da Faculdade Metropolitana da Amazônia (FAMAZ). Belém-Pará: Editora FAMAZ, Belém, 2012.

22. PIZZINATO A et al. A Integração Ensino-Serviço como Estratégia na Formação Profissional para o SUS. REVISTA BRASILEIRA DE EDUCAÇÃO MÉDICA. 36 (2); 170-177; 2012.

23. SCHEFFER M et al. Demografia Médica no Brasil: dados gerais e descrições de desigualdades. Relatório de pesquisa: 2011. São Paulo: Cremesp, 2011.

24. SHIMUZU HE, MOURA LM. As representações sociais do controle social em saúde: os avanços e entraves da participação social institucionalizada. Saúde Soc. São Paulo, 24(4);1180-1192, 2015.

25. SILVA AB et al. A assistência do enfermeiro da atenção básica ao adolescente com dependência química. R. Interd. 7(4);61-71. 2014.

26. VASCONCELOS ACF et al. An experience of teaching-service integration and change of professional practices: healthcare professionals speak out. Interface (Botucatu). 20 (56);147-58. 2016.

27. VENDRUSCOLO C et al. Integração ensino-serviço e sua interface no contexto da reorientação da formação na saúde. Interface - Comunicação, Saúde, Educação. 20(59); 15-25. 2016. 